\title{
A set of device generators for analogue and mixed-signal layout synthesis
}

\author{
J.-F. Naviner*, P. Loumeau**, O. Oliaei**, H. Petit**, \\ L. A. B. Naviner*, E. Melcher* \\ (*)DEE - CCT - Universidade Federal da Paraíba \\ Av. Aprígio Veloso, 882, Bodocongó \\ 58105-970 Campina Grande - Pb - Brazil \\ Tel. 5583 3101135 Fax 55 833101418 \\ email:naviner@dsc.ufpb.br
}

(**)Département Électronique

École Nationale Supérieure des Télécommunications

46, rue Barrault - 75634 Paris cedex 13 - France

Tel. 33145817843 Fax 331 45804036

\begin{abstract}
The layout synthesis of analog circuits remains today a complex task difficult to automate. Various strategies are used, from a completely full-custom design up to an automated synthesis taking into account analog constraints. One popular technique developed to help the analog layout synthesis is the use of procedural design of basic cells. These cells are afterwards assembled either manually or using other CAD tools. The device generators described in this paper are developed to enhance layout design productivity of analog cells. These generators are written in language Skill for the Opus-Cadence framework. They cover the most common devices: resistors, capacitors, inductors, various transistor shapes, differential pairs and current sources. The context and the objectives of this work, the development strategy and the main functionalities are presented. Finally, an example is given. These generators have been successfully used to design several circuits.
\end{abstract}

\section{Keywords}

CAD, Analog Layout Generation, Device Generators 


\section{INTRODUCTION}

During the last few years, the synthesis tools for analog or mixed-signal integrated circuits (ICs) have achieved a lot of significant improvements even if the commercial offer still remains reduced (Carley, 1996). The progresses are real at all levels: from layout cell synthesis up to macroblock synthesis including architecture or topology choice and circuit sizing. There are several factors increasing this tendency:

- More and more, today, a complete system integration on a chip becomes possible and necessary. To perform that, the implementation of analog and digital parts, sometimes also sensors or other devices on a same circuit is required. The very competitive market of microelectronics induces the need of methods and tools to design all parts of the system. A lack of tools for one part means much more human effort and a significant overcost for the final product.

- A large variety of mature tools is available for digital circuit design. Even if they are not always seen as ideal solutions from the designers point of view, they give a huge productivity gain which remains without counterpart in the analog domain where often a circuit design is still based on full custom methods using a very small set of CAD tools, mainly simulation and some analysis tools. This relatively mature state of tools for digital circuits has two consequences: Some previously digital CAD designers are now working to develop tools for analog or mixed-signal circuits. Some methods and algorithms earlier used to solve digital problems may often be adapted and partially re-used for other applications.

- Even if the number of basic components is much more reduced in analog circuits compared to digital ones, each component has to be considered with a much more precise description and modelization. Also, the number of parameters which may influence the performances or more generally the implemented function itself is big. A lot of degrees of freedom have to be managed. The first task is to acquire a detailed knowledge of the structures involved. Then, it becomes possible to develop methods and finally tools to help the design. The low cost computation power today available simplifies the development and use of specific algorithms to solve complex problems of analog design in a reasonable time.

One of the topics which is the object of a lot of efforts and improvements since a couple of years is the analog layout synthesis. In this area, two different problems are addressed: the cell layout synthesis which consists in the creation of masks from a transistor-level schematic under a set of constraints and the assembly of cells or macrocells, that is, the placement and routing of the whole analog circuit. This paper is focused on the study of analog cell synthesis.

The objective of analog cell layout synthesis is ideally to obtain masks completely according to the constraints and the desired performances. The mask 
layout should be as good as a full custom design realised by an expert designer, however with a much more shorter development time. To reach this objective, various strategies have been proposed. In the following section, the main approaches and their characteristics are presented.

Then, the analog cell layout generator approach is discussed with more details. A set of efficient cell layout generators has been developed. The layout strategy and the context of the development are presented. An example of a waffle shaped transistor generator is presented. This set of generators has been implemented in the Cadence Opus framework, using the parameter cells facilities.

\section{ANALOG LAYOUT STRATEGIES}

From the earlier attempts up to the most recent ones, the approaches have evolved from nearly digital strategies to analog performances oriented strategies. The first approaches developed were based on some analog device generators and placeand-route tools derived from the existing algorithms for digital circuits (Rijmenants, 1989). Another strategy was to develop a generator program for each different basic cell. This approach is interesting when only few changes (orientation, sizing, etc.) have to be realised in the layout. In such a case, the generation is fast and satisfactory (Kuhn, 1987). These tools are based on a rich device generator library. They require generally a considerable designer effort to perform the assembly of the generated structures in order to respect the analog constraints which are not directly included in the synthesis process. A strong interactivity is then necessary.

More recently, various tools have been developed in order to include analog characteristics into the synthesis of the layout (Cohn, 1994; Lampaert, 1995; Miliozzi, 1996). These systems are using also generators but only for very basic structures. With powerful algorithms, they construct more complex structures during the layout synthesis process, particularly by merging components. The objective is to keep all possibilities avoiding a priori choices without maintaining a rich set of complex generators. However, a limitation is that some optimised configurations can not be obtained. In some critical applications, the use of these optimised device layouts is important in order to reach the desired performances. The goal of theses tools is to complete in a fully automated way the optimised synthesis of an analog circuit from the definition at a higher level of all constraints and specifications of the design. One consequence is that interactivity should not be necessary. In fact, many algorithms used (simulated annealing for example) do not allow human interaction.

All strategies are therefore based on the use of generators, simple or complex depending mostly on the algorithms used for assembly. Various works recently described propose the use of sets of generators of medium complexity: optimised 
shaped transistors, basic active devices or passive components (Bruce, 1996; Owen, 1995). The work presented in this paper belongs to this strategy.

\section{PARAMETRIZED CELLS UNDER THE CADENCE-OPUS FRAMEWORK}

The set of generators has been developed with the facilities (Virtuoso Layout Editor - Parameterized cells) proposed in the Cadence Opus framework. The generators may be defined graphically or writing the corresponding code in the Skill language (Cadence). In this case, it was preferred to directly write in the Skill language because of the high level of parameterization necessary: none of the parameter values is hardcoded. The main transformations available for parameterized cells (pcells) are: stretch of polygons, repetition, inclusion of parameterized labels, inclusion of parameterized levels of masks, conditional inclusion of shapes, repetition along shapes, inheritance of parameters and parameterization of properties. The pcells may be instantiated as any other cell but the parameters may be specified each time.

\section{DEVELOPMENT STRATEGY}

\subsection{Technology definition}

One of the most important objective is the independence of the tool with respect to the technology evolutions. In practice, a change of a technology rule must not affect the code of the generators and must not require individual fitting or rewriting of each generator program. That is why the geometric rules and some electric rules were defined in a fully independent way: it was preferred to define a proper technological description without interference with the Opus technology file. This choice comes from two essential considerations:

- with proper rule definition, the generator program is immune with respect to some technology design-kit alterations and the requirements of other tools;

- moreover, a potentially future recoding in a more common language will be simpler if there is no strong link between the Opus structures and our system.

However, for major technology changes, the generator structures have sometimes to be changed.

The technology data are stored in a structure composed of basic parameters values and lists. The basic parameters are the minimum pitch and the name of the technology. The lists group the layers (group Layer) or geometrical or electrical rules of a same type. The groups of geometrical rules are Width, Space, Overlap and Intersection. The groups of electrical rules are Resistor and Capacitor. A group Other is used to described particular rules which do not correspond to the previous groups. 
A rule is a set of keywords with an associated value. The first keyword is the name of the group. The following keywords are usually symbolic names of layers. That is, for each mask layer level, is defined one or more symbolic name. For example, the following rules may be defined:

(Layer poly1 "poly")

'(Layer gate "poly")

These rules define two symbolic names of layers: polyl and gate. The definition of the symbolic names of layers has to be done first in order to use them for other kinds of rules. For instance, the overlap of polysilicon over a contact may be defined as following:

\section{'(Overlap polyl contact 0.30)}

Generally, only one or two symbolic layer names are necessary to characterise a rule. Sometimes, however, a greater number is required. The use of non symbolic names as keywords is also possible. This is particularly useful for the rules included in group Other. For example:

\section{(Other contactOnCapacitor $t$ )}

This rule specifies that the implementation of contacts on the top plate of a capacitor is allowed.

The formalism used to retrieve a value of a defined rule is similar to the one described above.

\subsection{Generator description}

A generator is a program which builds shapes, generally rectangles, from a set of user defined variables and a set of technology dependent predefined parameters. For example, for a MOS transistor generator, the channel width and length are user variables and the minimum width or the minimum length are technology dependent parameters.

Each generator is described in a separated file. They are all described with the following organisation:

- Declaration of the variables: Name and default value.

- Definition of local variables from the values of necessary technological rules (layers, geometrical and electrical rules).

- Computation of some local variables necessary to generate the shapes from the variables and the previous local variables.

- Generation of the shapes.

In some particular cases, a more complex structure has been used. For instance, a square capacitor array generator uses a set of small generators which produce different parts of the global structure. A program is therefore necessary to control the assembly of the parts of the array. For that the homogeneous array facilities provided by Opus are used. 


\subsection{Generator parameters}

The code of the generators is entirely defined using variables or parameters. There is no, direct reference to a numerical hard coded value. When a generator needs a technological parameter, first a set of rules associated with the generator is examined. All rules may be redefined for a particular generator. If not found, the rule is searched in the technology rule set. Finally, if not found, a default value is assumed.

Some data are only searched in the generator parameter set, for instance, the layers used. Sometimes, other parameters are defined to specify a fixed characteristic which may be different from a technology to another or from a desired configuration to another. For instance, a generator of squared capacitor arrays has been defined, with the possibility to allow or not the direct implementation of contacts on top of the upper plates.

\section{GENERATORS OVERVIEW}

The development of a generator library is a cumbersome task: The variety of generators must be large enough in order to cover the main needs of analog circuit design. Also, the generator set should offer some elaborated structures, to give a higher gain with respect to a fully manual design. However, the development of a complex generator may involve a loss of universality. That is why a lot of the automatic analog synthesis tools often use a very small set of device generators (Cohn, 1994). They consider that more complex configurations may be reached through the optimisation algorithms. Even so, some structures are really difficult to obtain automatically. That is why a set of generators should contain the basic structures and some others more elaborated ones. These are particularly important to optimise some electrical performances or to reduce area.

Today, the set of generators includes:

- folded transistors;

- differential pairs;

- current sources;

- waffle shaped transistors;

- capacitor arrays;

- inductors;

- resistors;

- contacts.

The possibly interesting shapes of a transistor are numerous. The waffle shaped configuration is well suited to implement very wide transistors (large channel 
width over length ratio). The structure offers a high channel width over length $(W / L)$ ratio per unit area while keeping low source, drain and gate resistors. Furthermore, the reduced areas of drain and source allow low junction capacitors on these terminals. Particularly, the side capacitance influence is low. A typical waffle-shaped transistor is represented on the figure below (Figure 1): the gate is subdivided in segments distributed horizontally and vertically in order to form a grid. The source and drain connection points take place between the gate segments. They are connected by diagonal metal lines.

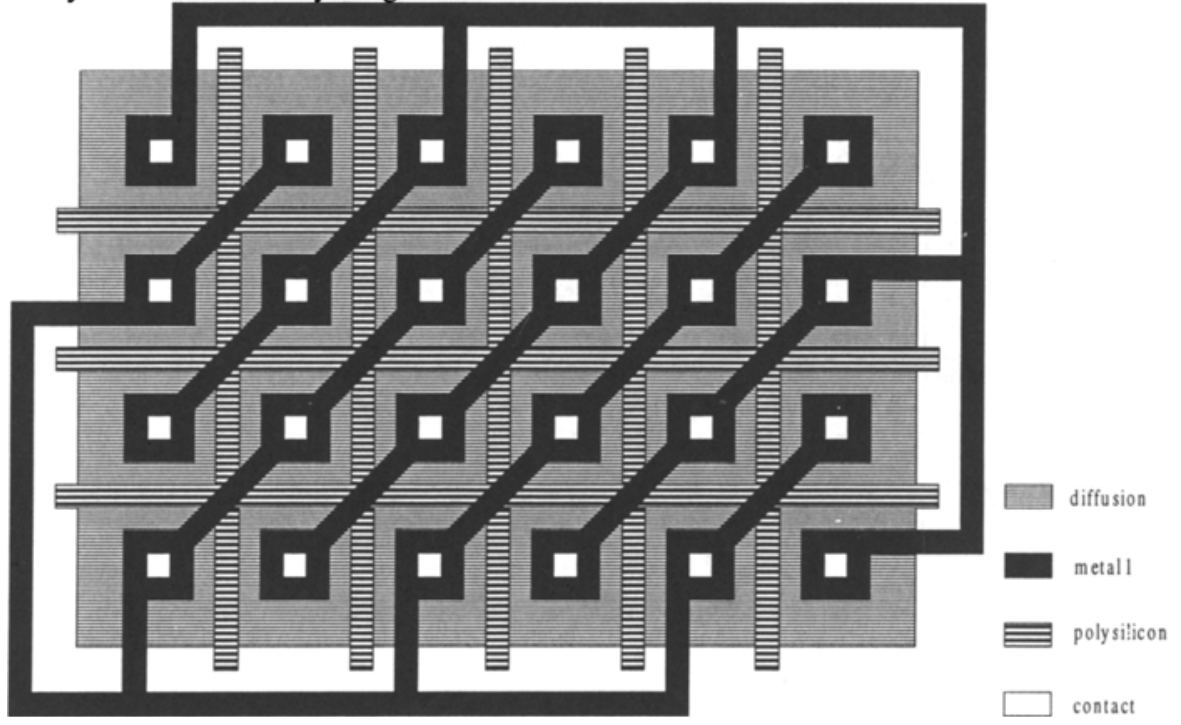

Figure 1 Waffle shaped transistor

A drawback of this structure is that the connections between drains and the connections between sources are necessarily realised traversing the gate segments which induces an increase of parasitic capacitance between the source and the gate and between the drain and the gate. This parasitic capacitance may cause problems for some applications. An other limitation of this structure compared with simpler ones is that there is no possibility to merge or to realise interdigitized transistors. It may be noticed also that this structure is not well characterised: because of the crossing of gate segments, the total channel width of the transistor is difficult to evaluate. As long as there does not exist an accurate model to compute the width of the transistor, the use of this structure will be restricted to applications requiring a low precision on the characteristics. The structure may be used also in digital circuits to implement buffers.

In this section, is described how to design the component and the main characteristics of the developed generator.

The following notations are used (Figure 2):

- W total channel width;

- L channel length; 
- hg number of horizontal gate segments;

- $v g$ number of vertical gate segments;

- $d g \quad$ distance between two parallel gates;

- $h w$ horizontal external width of source or drain;

- $v w \quad$ vertical external width of source or drain;

- gc factor to take into account the crossing of gate segments ( $0 \leq \mathrm{gc}$ $\leq 1)$

- $d d$ coefficient to compute distances with diagonal connections (dd > $\sqrt{2})$.

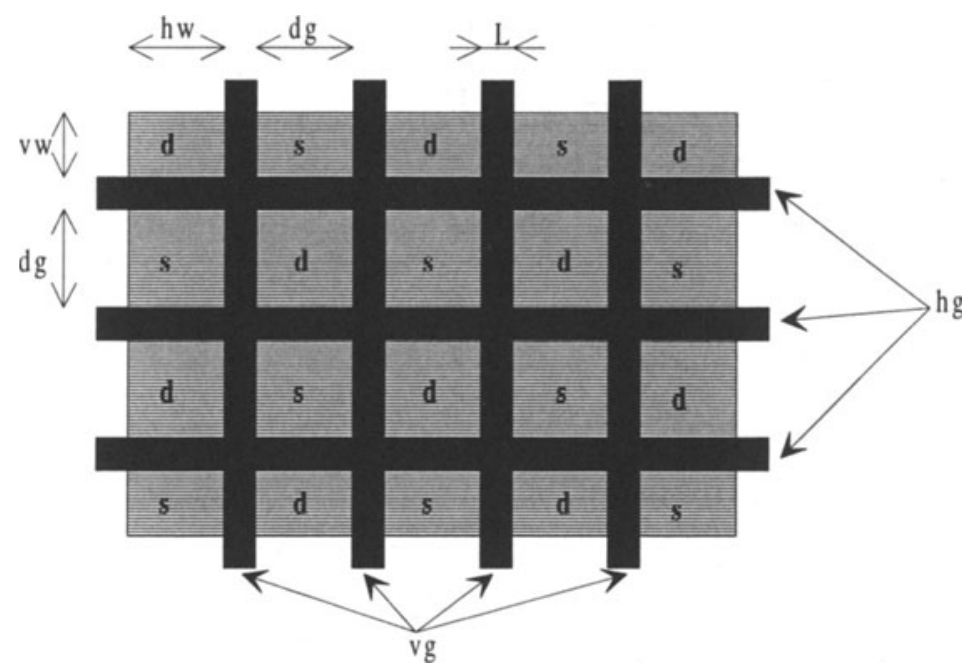

Figure 2 Notations

\subsection{Distance between gate segments}

It is interesting to minimise the distance between gate segments in order to maximise the gate density and to minimise the source and drain areas. Hence, the parameter $\mathrm{dg}$ is computed from the geometric rules of the technology. In most cases, the diagonal constraint on metall determines its value.

\subsection{Total width of the transistor}

It is assumed in this section that the number of horizontal and vertical gate segments are parameters. The expression of the total width as a function of these parameters is deduced.

For the core of the transistor (without the external channels):

$W_{\text {core }}=(v g-1) \times h g \times(d g+g c \times L)+(h g-1) \times v g \times(d g+g c \times L)$

For the external channels:

$W_{\text {extem }}=v g \times(2 \times v w+g c \times L)+h g \times(2 \times h w+g c \times L)$

$W_{\text {toul }}=W_{\text {core }}+W_{\text {extern }}$ 


\subsection{Horizontal and vertical numbers of gate segments}

The external channel widths $h w$ and $v w$ admit a lower bound $w e_{\min }$. That is determined by the area necessary to implement a contact on an external source or drain.

In order to determine the horizontal and vertical numbers of gate segments, the previous equation of $W_{\text {total }}$ substituting $h w$ and $v w$ by $w e_{\text {min }}$ is used. A set of couples $(h g, v g)$ is determined such that:

$W\left(h g, v g, w e_{\min }, w e_{\min }\right) \leq W_{\text {desired }}$

$W\left(h g+1, v g, w e_{\text {min }}, w e_{\text {min }}\right)>W_{\text {desired }}$

$W\left(h g, v g+1, w e_{\text {min }}, w e_{\text {min }}\right)>W_{\text {desired }}$

From this set, a specified form factor may be used to choose gate segment numbers. Then, $h w$ and $v w$ are adjusted to obtain $W_{\text {desired }}=W_{\text {toull }}$.

\subsection{Waffle shaped transistor generator}

Currently, the implemented waffle shaped transistor generator is defined from the following parameters:

- length

- horFoldFactor

- verFoldFactor

- lateralHorWidth drain;

- lateralVerWidth

From these parameters, the total width of the transistor channel is computed. Also, the total area, perimeter of source and drain and parasitic capacitors are calculated.

\section{CIRCUITS DESIGNED WITH THE GENERATORS}

Various circuits have been designed using the set of generators described in this paper (Porte, 1994; Oliaei, 1996). Some of them are shortly described below.

- A circuit was designed to implement an Operational Transconductance Amplifier with differential structure. The layout design was easily performed based on a symmetrical axis. Generators were used for differential pair, but also for other structures like current mirrors, output stage and common mode feedback.

- A p-n junction-based precision temperature transducer circuit was integrated. All analog parts of the circuit were designed using the generators (resistors, operational amplifiers, switches, capacitor arrays). This circuit is currently in fabrication (Freire, 1996). 
- A switched current sigma delta modulator has been implemented. An accurate relationship between different currents is essential in this type of circuits. Generators were used to realise track and hold, integrators and comparators of currents. The circuit is based on switched-current class $\mathrm{AB}$ sigma delta modulator (Oliaei, 1997a).

- A current multiplier circuit has been designed. The circuit layout is based on a symmetrical axis. Cascoded current mirrors are particularly used. This is a CMOS class AB current multiplier (Oliaei, 1997b).

- Recently, a circuit was designed to test the realisation of inductors in CMOS technology. Generators were used to realise different inductors to test the feasibility for radiocommunication applications.

In future, an advanced version of the generator set will be associated to a sigmadelta switched current structure synthesis tool currently in study.

\section{FUTURE WORKS}

The principal objective of development is to constitute a complete system for analog cell synthesis from specifications to the final layout.

Currently, the generators are being rewritten in $\mathrm{C}$ language. The following improvements are incorporated:

- two representation levels are used: a layout level and an abstract level. The abstract level contains a subset of geometrical and electrical data useful for the placement program.

- when it is not already implemented, parasitics are calculated.

The entries of the analog synthesis tool will be the technology data, user specifications and a netlist generated with COMDIAC, a tool developed at the École Nationale Supérieure des Télécommunications. From a set of user specifications and technology data, COMDIAC produces a sized netlist with some other results: operating-point tensions and currents, obtained specifications and limits.

From the results produced by COMDIAC, the interesting shapes at the abstract level will be generated for each device of the netlist.

At the same time, a first selection of potentially interesting topologies will be performed. This first placement will be based only on symmetries, weighted matching properties of elements and eventually some user constraints. At this phase, the user may interact to eliminate or impose topologies.

Then, each selected topology will be analysed using the abstract level representation of devices, an evaluation of routing and the electrical characteristics. The various orientations of the devices will be tested. As in (Lampaert, 1995), the sensitivities will be used in order to evaluate the degradation of performances induced by a topology. A subset of shapes with the best results will be kept. 
Finally, each selected shape will be routed and analysed again in order to select the best one.

In the proposed strategy described above, it will be tried to avoid algorithms which include random choices as it occurs with simulated annealing or some genetic algorithms.

\section{CONCLUSION}

A set of layout generators for basic devices has been presented. Written in Skill for the Cadence-Opus framework, they are easily adaptable for compatible CMOS technologies by redefining a parameter file. They have been used successfully to design the layout of various analog integrated circuits in a double-polysilicon, double-metal CMOS technology. The use of the generators reduces the time required to layout a circuit and allows to experiment rapidly various configurations in order to obtain the best performances. Taking advantage of the formalism defined, new generators may be created easily. Rewritten in C language, these generators will be a base for a analog cell synthesis tool and for a sigma-delta switched-current structure synthesis tool.

\section{REFERENCES}

Bruce J.D., Li H.W., Dallabetta M.J., Baker R.J. (1996) Analog Layout Using ALAS!. IEEE JSSC, Vol. 31, No 2, pp. 271-274, february 1996.

Cadence - Opus Framework On-Line Documentation.

Carley L.R., Gielen G.G.E., Rutenbar R.A., Sansen W.M.C. (1996) Synthesis Tools for Mixed-Signal ICs: Progress on Frontend and Backend Strategies, in proceedings of the 33rd Design Automation Conference.

Cohn J.M., Garrod D.J., Rutenbar R.A., Carley L.R., (1994) Analog Device-Level Layout Automation. Kluwer Academic Publishers.

Freire R.C.S., Deep G.S., Naviner J.-F., Loumeau P., (1996) A p-n junction-based precision temperature transducer integrated circuit, in proceedings of the International Workshop on Thermal Investigations of Ics and Microstructures, setember 1996.

Kuhn J. (1987) Analog Module Generators for Silicon Compilation. VLSI System Design, May 1987.

Lampaert K., Gielen G., Sansen W.M., (1995) A performance-driven placement tool for analog integrated circuits. IEEE JSSC, Vol. 30, N'7, pp. 773-780, July 1995.

Miliozzi P., Vassiliou I., Charbon E., Malavasi E., Sangiovanni-Vincentelli A.L. (1996) Use of Sensitivities and Generalised Substrate Models in Mixed-Signal IC Design, in proceedings of the 33rd Design Automation Conference. 
Oliaei O., Loumeau P. (1996), A low-input resistance class AB CMOS current conveyor, in proceedings of the 39th Midwest Symposium on Circuits and Systems, august 1996.

Oliaei O., Loumeau P. and Aboushady H. (1997a) A Switched-Current Class AB Sigma-Delta Modulator, to be published at ISCAS, July 1997.

Oliaei O. and Loumeau P. (1997b) A CMOS Class AB Current-Multiplier, to be published at ISCAS, july 1997.

Owen B.R. (1995) BALLISTIC: An Analog Layout Language - Beta+ Version Reference Document. University of Toronto.

Porte J., Yang F., Azadet K., Naviner J.F. (1994) Continuous-Time Current-Mode Filters Using Normal Biquad Structure, in proceedings of the 37th Midwest Symposium on Circuits and Systems, pp. 994-997, august 1994.

Rijmenants J., et al. (1989) ILAC: An automated layout tool for analog CMOS circuits. IEEE JSSC, Vol. 24, n 4, pp. 417-425, April 1989. 